\title{
Opening of Exocycle of the Methyl Pheophorbide a 13(2)-Amide Derivatives in a Water-Acetone Solution in the Presence Hydrochloric Acid
}

\author{
D. V. Belykh, ${ }^{@}$ and I. S. Khudyaeva \\ Federal State Budget Organization of Science Institute of Chemistry, Komi Science Centre, Ural Division RAS, 167000 \\ Syktyvkar, Russia \\ ${ }^{\circledR}$ Corresponding author E-mail: belykh-dv@mail.ru
}

\begin{abstract}
It has been shown that under the action of water on methyl pheophorbide a 13(2)-amide derivatives in acidic conditions (mixture of concentrated hydrochloric acid and acetone), in addition to hydrolyzing of the ester group of the substituent at position 17, exocycle opening with the formation of the corresponding chlorin $e_{6} 15$-amide derivatives occurs. The possibility of exocycle opening seems to be caused by its strength due to the presence of a relatively bulky amide substituent, as well as to the protonation of the amide group, which facilitates the rupture of the C(13(1))-C(13(2)) bond.
\end{abstract}

Keywords: Methylpheophorbide $a$, chlorin $e_{6}$, amides, hydrolysis, exocycle opening, ester group.

\section{Размыкание экзоцикла 13(2)-амидных производных метилфеофорбида $a$ в водно-ацетоновом растворе в присутствии ХлоровоАОРОАНОЙ КИСАОТЫ}

\author{
А. В. Белых, ${ }^{\circledR}$ И. С. Худяева \\ Институт химии Коми научного ијентра Уральского отделения Российской академии наук, 167000 Сыктывкар, \\ Россия \\ ${ }^{\circledR}$ E-mail: belykh-dv@mail.ru
}

\begin{abstract}
Показано, что при действии воды на 13(2)-амиднье производнье метилфеофорбида а в кисльх условиях (смесь кониентрированной соляной кислоты с ацетоном) помимо гидролиза сложноэфирной группь заместителя в положении 17 происходит размыкание экзоцикла с образованием соответствующих 15-амидных производных хлорина е $e_{6}$ Возможность размыкания экзоцикла, по всей видимости, связана с его напряженностью вследствие наличия относительно объемного амидного заместителя, а также с протонированием амидной группь, способствующим разрыву связи С(13(1))-С(13(2)).
\end{abstract}

Ключевые слова: Метилфеофорбид $a$, хлорин $e_{6}$, амиды, гидролиз, размыкание экзоцикла, сложноэфирная группа. 
Chlorophyll $a$ and its derivatives, especially methylpheophorbide $a$, are a promising basis for the synthesis of biologically active substances, such as photosensitizers for photodynamic therapy of cance ${ }^{[1-4]}$ and microbial diseases ${ }^{[4,5]}$ diagnostic drugs for oncology, ${ }^{[4]}$ hybrid antioxidants $^{[6-9]}$ and others. The conjugation of a chlorin macrocycle with a fragment of the desired structure allows to assign the necessary properties for the molecule as a whole. We have previously shown that the amidation of the ester group at position 13(2) of the methyl pheophorbide $a$ exocycle is a convenient way of forming an amide bond having a significant synthetic potential. ${ }^{[6-14]}$ The use of this reaction is a convenient way of synthesizing the conjugates of methylpheophorbide $a$ and its analogs with terpenophenols, ${ }^{[6-9]}$ introducing bulk alkyl substituents, ${ }^{[10,11,13]}$ as well as obtaining dimeric and trimeric chlorophyll $a$ derivatives. ${ }^{[12,14]}$ The hydrolysis of the 17-substituent ester group in such conjugates is of interest from the viewpoint of hydrophilizing the resulting derivatives, as well as further chemical modification using the carboxyl group reactions. Acid catalysis (for example, reaction in a mixture of acetone with concentrated hydrochloric acid) is suitable for 17-substituent ester group hydrolysis. ${ }^{[15]}$ These conditions have been successfully used for many chlorophyll $a$ derivatives, such as pheophytin $a$ (1), methyl pheophorbide $a$ (2), methylpyropheophorbide $a$ (3), etc. (Scheme 1). The reaction occurs chemoselectively and with good yields of the target carboxylic derivatives. ${ }^{[7,14,15]}$ To determine the possibility of this reaction application to the methylpheophorbide $a$ 13-amide derivatives the conversion of analogous simple derivatives (4-8) (Scheme 2) in acetone with concentrated hydrochloric acid were studied in this paper.

It was found that in water-acetone solution at the presence of hydrochloric acid amide derivatives (4-8) the exocy- cle opening with corresponding chlorin $e_{6} 15$-amide derivatives (9-13) formation occurs in addition to hydrolysis of the ester group of the substituent at position 17 (Scheme 2). The structure of the compounds obtained 9-13 was established based on IR, electronic (UV-Vis) and NMR spectroscopy, as well as mass spectrometry data. There is no singlet corresponding to the proton at position 13(2) and doublets corresponding to protons of the methylene group of the substituent at position 15 formed as a result of the exocycle opening are observed in ${ }^{1} \mathrm{H}$ NMR spectra of the compounds obtained 9-13. In IR spectra of 9-13, in comparison with the spectra of starting compounds $\mathbf{4 - 8}$, there is no absorption band corresponding to the carbonyl group at position 13(1), that also indicates the exocycle opening. The presence of three three-proton singlets (instead of four such singlets in the spectrum of the starting compounds) in the ${ }^{1} \mathrm{H}$ NMR spectra shows that the ester group at position 17 was hydrolyzed. The broadened two-proton singlet about 13 ppm corresponds to the protons of the two carboxyl groups formed in these processes. The position of intra-cyclic NH-protons signals also confirms the exocycle opening: the signals of both intra-cyclic $\mathrm{NH}$ protons are observed in the region of chemical shifts negative values (from -1.6 to $-2.5 \mathrm{ppm}$ ), while at presence of an exocycle the signal of the pyrrole ring III NH proton is observed at positive chemical shift values (from 0.6 to $0.3 \mathrm{ppm}$ ). In the mass spectra (ESI) of the compounds obtained the peaks corresponding to protonated molecular ions $\mathbf{9 - 1 3}$ are observed that is in agreement with the IR and ${ }^{1} \mathrm{H}$ NMR spectroscopy data.

The reaction found is of a great preparative and theoretical interest. It can be used to prepare dicarboxy-chlorins with unstable in an alkaline medium substituents. The formation of chlorin $e_{6}$ 15-amide derivatives from methylpheophorbide a 13(2)-amide derivatives proceeds

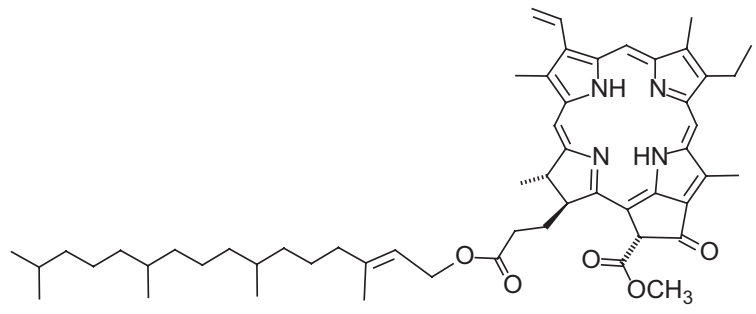

(1)

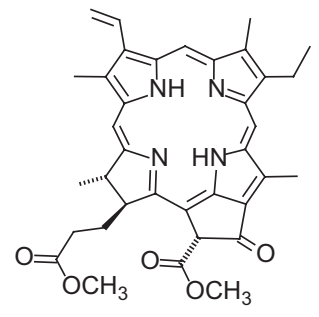

(2)

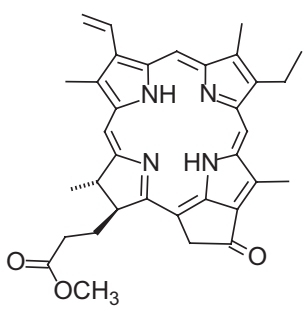

(3)

Scheme 1.

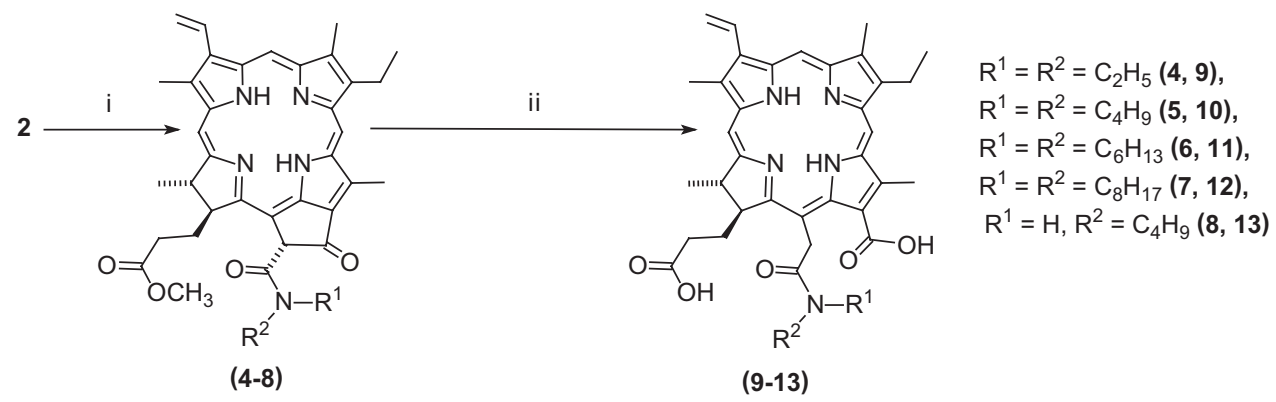

Scheme 2. i: toluene, reflux 4, $\mathbf{8},{ }^{[13]} \mathbf{5}-\mathbf{7}^{[10]}$; ii: $\mathrm{H}_{2} \mathrm{O}-\mathrm{HCl} /$ acetone, $12 \mathrm{~h}$ for conversion of $\mathbf{5}-\mathbf{7}$ to $\mathbf{1 0}-\mathbf{1 2}$, respectively, $144 \mathrm{~h}$ for conversion of 4 and 8 to 9 and 13 , respectively. 


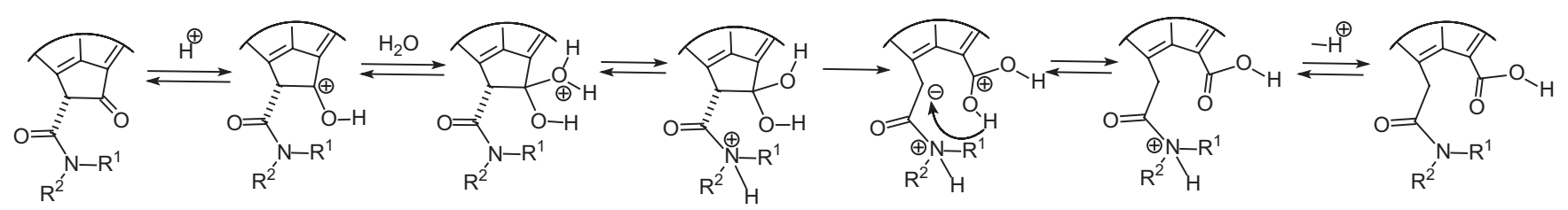

Scheme 3.

in an acidic medium, where the only nucleophile can recover the exocycle is the water molecule. The elucidations of such reactions possible reasons supplement the information on the chemical properties of such derivatives significantly. It is known that the exocycle is not opened under similar conditions in the case of chlorophyll $a$ derivatives having an ester group (such as pheophytin $a$ (1), methyl pheophorbide $a(2)$, etc.) at position 13(2). The fact that the exocycle opening occurs only in the case of amide derivatives allows to suggest that this reaction is promoted by the protonation of the 13(2)-amide group, that stimulates the breaking of the $\mathrm{C}(13(1))-\mathrm{C}(13(2))$ bond (Scheme 3). Similar protonation of the ester group occurs to a considerable lesser extent, that increases the stability of the exocycle under acidic conditions. This is also facilitated by larger size of the amide group (especially tertiary) compared with the ester that enhances the exocycle tension. The small size of the water molecule does not lead to steric hindrances for the nucleophilic attack of the carbonyl carbon atom at position 13(1), and protonation of the oxygen atom of this keto group can facilitate the opening of the exocycle (Scheme 3 ).

Thus, we have shown that in aqueous-acetone solution at presence of hydrochloric acid, 13(2)-amide derivatives (4-8) exocycle is opened with the formation of the corresponding chlorin $e_{6} 15$-amide derivatives $(\mathbf{9 - 1 3})$ in addition to the substituent at position 17 ester group hydrolysis (Scheme 2). The possibility of the exocycle opening seems to be due to its strength and presence of a relatively bulky amide substituent as well as to the amide group protonation that facilitates the breaking of the $\mathrm{C}(13(1))-\mathrm{C}(13(2))$ bond (Scheme 3).

\section{Experimental}

IR spectra were recorded on a Shimadzu IR Prestige 21 FTIR spectrometer in $\mathrm{KBr}$ tablets. Electronic spectra were recorded on a Shimadzu UV-1700 spectrometer in quartz cuvettes of $10 \mathrm{~mm}$ thickness (chloroform comparison sample). ${ }^{1} \mathrm{H}$ NMR spectra were recorded on a Bruker Avance II spectrometer (operating frequency $300 \mathrm{MHz}$ ) for solutions of substances in DMSO- $d$. ESI mass spectra were recorded on a Thermo Finnigan LCQ Fleet. The reaction was monitored by TLC on Sorbfil plates. Silica gel $70 / 230 \mu$ (Alfa Aesar) was used for column chromatography.

The methyl pheophorbide $a$ 13(2)-amides (4-8) were dissolved in a mixture of acetone $(10-20 \mathrm{ml})$ with hydrochloric acid $(0.5-1.0 \mathrm{ml})$ and left for 12 hours $(\mathbf{5}-7)$ or 6 days $(\mathbf{4}, \mathbf{8})$. Chloroform was then added and the acid and acetone were washed with water until a neutral reaction of the washings. The resulting solution was dried over anhydrous sodium sulfate and evaporated under reduced pressure at a temperature of $40-50{ }^{\circ} \mathrm{C}$. The residue after evaporation was chromatographed on silica gel (elution with a mixture of $\mathrm{CCl}_{4}$ :acetone in proportions from $50: 1$ to $1: 1$ by volume). The eluates containing the main product were evaporated under reduced pressure.

Chlorin $e_{6}$ 15(2)-N,N-diethylamide (9). $24.5 \mathrm{mg}$ (44\%) of compound 9 as a dark green crystalline powder were obtained from $55.1 \mathrm{mg}$ of compound 4. $\mathrm{m} / z$ (ESI) calculated for $[\mathrm{MH}]^{+}$ $\left(\mathrm{C}_{38} \mathrm{H}_{45} \mathrm{~N}_{5} \mathrm{O}_{5}\right): 652.3$, found: 652.4. IR $(\mathrm{KBr}) v_{\max } \mathrm{cm}^{-1}: 3298$ $(v(\mathrm{NH})), 2965,2930,2870,2737,2592\left(v(\mathrm{C}-\mathrm{H}) \mathrm{CH}_{3}, \mathrm{CH}_{2}\right), 1726$ $(v(\mathrm{C}=\mathrm{O}) \mathrm{COOH}), 1603$ ("Amide-I», "Chlorin band»). UV-Vis $\left(\mathrm{CHCl}_{3}\right) \lambda_{\text {max }} \mathrm{nm}$ 663.5, 608.5, 558.0, 531.0, 501.5, 403.5. ${ }^{1} \mathrm{H}$ NMR (DMSO- $\left.d_{6}, 300 \mathrm{MHz}\right) \delta_{\mathrm{H}}$ ppm: 13.93 (1H, br.s, 13(1)-COOH), $12.19(1 \mathrm{H}$, br.s, $17(3)-\mathrm{COOH}), 9.79\left(1 \mathrm{H}, \mathrm{s}, \mathrm{H}^{10}\right), 9.73\left(1 \mathrm{H}, \mathrm{s}, \mathrm{H}^{5}\right)$, $9.11\left(1 \mathrm{H}, \mathrm{s}, \mathrm{H}^{20}\right), 8.34\left(1 \mathrm{H}\right.$, dd $J=18.0$ and $\left.12.0 \mathrm{~Hz}, 3-\mathrm{CH}=\mathrm{CH}_{2}\right)$, $6.48\left(1 \mathrm{H}, \mathrm{d} J=18.0 \mathrm{~Hz}, 3-\mathrm{CH}=\mathrm{CH} H_{\text {trans }}\right), 6.19(1 \mathrm{H}, \mathrm{d} J=11.9 \mathrm{~Hz}$, $\left.3-\mathrm{CH}=\mathrm{CH}_{\text {cis }}\right), 5.48\left(2 \mathrm{H}, \mathrm{s}, \mathrm{H}^{15(1)}{ }_{\mathrm{A}}, \mathrm{H}^{\text {trans(1) }}{ }_{\mathrm{B}}\right), 4.61(1 \mathrm{H}, \mathrm{q} J=9.0 \mathrm{~Hz}$, $\left.\mathrm{H}^{18}\right), 4.41\left(1 \mathrm{H}\right.$, br.d $\left.J=9.0 \mathrm{~Hz}, \mathrm{H}^{17}\right), 3.92-3.77\left(2 \mathrm{H}, \mathrm{m}, 8-\mathrm{CH}_{2} \mathrm{CH}_{3}\right)$, $3.85\left(3 \mathrm{H}, \mathrm{s}, 12-\mathrm{CH}_{3}\right), 3.59\left(3 \mathrm{H}, \mathrm{s}, 2-\mathrm{CH}_{3}\right), 3.55\left(3 \mathrm{H}, \mathrm{s}, 7-\mathrm{CH}_{3}\right)$, 2.40-2.06 (4H, m, 17- $\left.\mathrm{CH}_{2} \mathrm{CH}_{2} \mathrm{COOCH}_{3}\right), 1.78-1.61(6 \mathrm{H}, \mathrm{m}$, $\left.18-\mathrm{CH}_{3}, 8-\mathrm{CH}_{2} \mathrm{CH}_{3}\right), 1.60-1.46\left(4 \mathrm{H}, \mathrm{m}, 15(2)-\mathrm{CON}\left(\mathrm{CH}_{2} \mathrm{CH}_{3}\right)_{2}\right)$, $1.26-0.97$ (6H, m, 15(2)- $\left.\mathrm{CON}\left(\mathrm{CH}_{2} \mathrm{CH}_{3}\right)_{2}\right),-1.64$ (1H, br.s, I-NH), -1.93 (1H, br.s, III-NH).

Chlorin $e_{6}$ 15(2)-N,N-dibutylamide (10). $40.8 \mathrm{mg}$ (42\%) of compound $\mathbf{1 0}$ as a dark green crystalline powder were obtained from $96.2 \mathrm{mg}$ of compound 5. $\mathrm{m} / \mathrm{z}$ (ESI) calculated for $[\mathrm{MH}]^{+}$ $\left(\mathrm{C}_{42} \mathrm{H}_{53} \mathrm{~N}_{5} \mathrm{O}_{5}\right)$ : 708.4, found: 708.4. IR $(\mathrm{KBr}) v_{\max } \mathrm{cm}^{-1}: 3298$ $(v(\mathrm{NH})), 2959,2930,2868,2731,2579\left(v(\mathrm{C}-\mathrm{H}) \mathrm{CH}_{3}, \mathrm{CH}_{2}\right), 1726$ $(v(\mathrm{C}=\mathrm{O}) \mathrm{COOH}), 1603$ («Amide-I», «Chlorin band»). UV-Vis $\left(\mathrm{CHCl}_{3}\right) \lambda_{\max } \mathrm{nm}: 663.5,607.5,556.5,531.0,502.0,404.0 .{ }^{1} \mathrm{H} \mathrm{NMR}$ (DMSO- $\left.d_{6}, 300 \mathrm{MHz}\right) \delta_{\mathrm{H}}$ ppm: $12.35(2 \mathrm{H}$, br.s, 13(1)-COOH and 17(3)-COOH), $9.77\left(1 \mathrm{H}, \mathrm{s}, \mathrm{H}^{10}\right), 9.73\left(1 \mathrm{H}, \mathrm{s}, \mathrm{H}^{5}\right), 9.10(1 \mathrm{H}, \mathrm{s}$, $\left.\mathrm{H}^{20}\right), 8.36\left(1 \mathrm{H}\right.$, dd $J=18.3$ and $\left.11.7 \mathrm{~Hz}, 3-\mathrm{CH}=\mathrm{CH}_{2}\right), 6.47(1 \mathrm{H}, \mathrm{d}$ $\left.J=18.0 \mathrm{~Hz}, 3-\mathrm{CH}=\mathrm{CH} H_{\text {trans }}\right), 6.19\left(1 \mathrm{H}, \mathrm{d} J=11.4 \mathrm{~Hz}, 3-\mathrm{CH}=\mathrm{CH} H_{\text {cis }}\right)$, $5.86-5.63\left(1 \mathrm{H}, \mathrm{m}, \mathrm{H}^{15(1)}{ }_{\mathrm{A}}\right), 5.39\left(1 \mathrm{H}, \mathrm{d} J=17.9 \mathrm{~Hz}, \mathrm{H}^{15(1)}{ }_{\mathrm{B}}\right), 4.60$ $\left(1 \mathrm{H}, \mathrm{q} J=6.6 \mathrm{~Hz}, \mathrm{H}^{18}\right), 4.32\left(1 \mathrm{H}\right.$, br.d $\left.J=10.3 \mathrm{~Hz}, \mathrm{H}^{17}\right), 3.85(2 \mathrm{H}, \mathrm{q}$ $\left.J=7.3 \mathrm{~Hz}, 8-\mathrm{CH}_{2} \mathrm{CH}_{3}\right), 3.56\left(3 \mathrm{H}, \mathrm{s}, 12-\mathrm{CH}_{3}\right), 3.54\left(3 \mathrm{H}, \mathrm{s}, 2-\mathrm{CH}_{3}\right)$, $3.35\left(3 \mathrm{H}, \mathrm{s}, 7-\mathrm{CH}_{3}\right), 2.31-1.86\left(4 \mathrm{H}, \mathrm{m}, 17-\mathrm{CH}_{2} \mathrm{CH}_{2} \mathrm{COOCH}_{3}\right)$, $1.71\left(3 \mathrm{H}, \mathrm{t} J=7.3 \mathrm{~Hz}, 8-\mathrm{CH}_{2} \mathrm{CH}_{3}\right), 1.65\left(3 \mathrm{H}, \mathrm{d} J=7.0 \mathrm{~Hz}, 18-\mathrm{CH}_{3}\right)$, $1.62-1.15\left(12 \mathrm{H}, \mathrm{m}, 15(2)-\mathrm{CON}\left(\left(\mathrm{CH}_{2}\right)_{3} \mathrm{CH}_{3}\right)_{2}\right), 1.07$ and 0.87 (both $3 \mathrm{H}$, both t $\left.\left.J=8.3 \mathrm{~Hz}, 15(2)-\mathrm{CON}\left(\mathrm{CH}_{2}\right)_{3} \mathrm{CH}_{3}\right)_{2}\right),-1.77$ (1H, br.s, I-NH), -2.20 (1H, br.s, III-NH).

Chlorin $e_{6}$ 15(2)- $N, N$-dihexylamide (11). $4.1 \mathrm{mg}$ (28\%) of compound $\mathbf{1 1}$ as a dark green crystalline powder were obtained from $14.8 \mathrm{mg}$ of compound $6 . \mathrm{m} / \mathrm{z}$ (ESI) calculated for $[\mathrm{MH}]^{+}$ $\left(\mathrm{C}_{46} \mathrm{H}_{61} \mathrm{~N}_{5} \mathrm{O}_{5}\right): 764.5$, found: 764.7. IR (KBr) $v_{\max } \mathrm{cm}^{-1}: 3300$ (v(NH)), 2957, 2926, 2858, 2731, $2669\left(v(\mathrm{C}-\mathrm{H}) \mathrm{CH}_{3}, \mathrm{CH}_{2}\right), 1738$, $1705(v(\mathrm{C}=\mathrm{O}) \mathrm{COOH}), 1603$ ( Amide-I», «Chlorin band»). UV-Vis $\left(\mathrm{CHCl}_{3}\right) \lambda_{\max } \mathrm{nm}: 664.0,608.5,556.5,531.0,502.0,404.0 .{ }^{1} \mathrm{H} \mathrm{NMR}$ (DMSO- $\left.d_{6}, 300 \mathrm{MHz}\right) \delta_{\mathrm{H}}$ ppm: $13.86(2 \mathrm{H}$, br.s, 13(1)-COOH), $12.11\left(1 \mathrm{H}\right.$, br.s, 17(3)-COOH), $9.79\left(1 \mathrm{H}, \mathrm{s}, \mathrm{H}^{10}\right), 9.70\left(1 \mathrm{H}, \mathrm{s}, \mathrm{H}^{5}\right)$, $9.12\left(1 \mathrm{H}, \mathrm{s}, \mathrm{H}^{20}\right), 8.37\left(1 \mathrm{H}, \mathrm{dd} J=18.0\right.$ and $\left.12.0 \mathrm{~Hz}, 3-\mathrm{C} H=\mathrm{CH}_{2}\right)$, $6.47\left(1 \mathrm{H}, \mathrm{d} J=18.0 \mathrm{~Hz}, 3-\mathrm{CH}=\mathrm{CH} H_{\text {trans }}\right), 6.18(1 \mathrm{H}, \mathrm{d} J=11.9 \mathrm{~Hz}$, $\left.3-\mathrm{CH}=\mathrm{CH} H_{\mathrm{cis}}\right), 6.11-5.76\left(1 \mathrm{H}, \mathrm{m}, \mathrm{H}^{15(1)}\right), 5.48-5.14\left(1 \mathrm{H}, \mathrm{m}, \mathrm{H}^{15(1)}\right.$ $\left.{ }_{\mathrm{B}}\right), 4.69-4.47^{\mathrm{cis}}\left(1 \mathrm{H}, \mathrm{m}, \mathrm{H}^{18}\right), 4.42-4.22\left(1 \mathrm{H}, \mathrm{m}, \mathrm{H}^{17}\right), 3.91-3.76$ $\left(2 \mathrm{H}, \mathrm{m}, 8-\mathrm{CH}_{2} \mathrm{CH}_{3}\right), 3.56\left(3 \mathrm{H}, \mathrm{s}, 12-\mathrm{CH}_{3}\right), 3.52\left(6 \mathrm{H}, \mathrm{s}, 2-\mathrm{CH}_{3}\right.$, 7- $\left.\mathrm{CH}_{3}\right), 2.40-2.02\left(4 \mathrm{H}, \mathrm{m}, 17-\mathrm{CH}_{2} \mathrm{CH}_{2} \mathrm{COOCH}_{3}\right), 1.76-1.62(6 \mathrm{H}$, 
$\left.\mathrm{m}, 18-\mathrm{CH}_{3}, 8-\mathrm{CH}_{2} \mathrm{CH}_{3}\right), 1.57-1.01\left(26 \mathrm{H}, \mathrm{m}, 15(2)-\mathrm{CON}\left(\mathrm{C}_{6} \mathrm{H}_{13}\right)_{2}\right)$, -1.85 (1H, br.s, I-NH), -2.39 (1H, br.s, III-NH).

Chlorin $e_{6}$ 15(2)-N,N-dioctylamide (12). $3.2 \mathrm{mg}$ (31 \%) of compound $\mathbf{1 2}$ as a dark green crystalline powder were obtained from $10.6 \mathrm{mg}$ of compound 7. $\mathrm{m} / \mathrm{z}$ (ESI) calculated for $[\mathrm{MH}]^{+}$ $\left(\mathrm{C}_{50} \mathrm{H}_{69} \mathrm{~N}_{5} \mathrm{O}_{5}\right)$ : 821.0, found: 821.1. IR ( KBr) $v_{\max } \mathrm{cm}^{-1}: 3298(\mathrm{v}(\mathrm{NH}))$, $2957,2926,2864,2731,2594\left(v(\mathrm{C}-\mathrm{H}) \mathrm{CH}_{3}, \mathrm{CH}_{2}\right), 1738,1710(v(\mathrm{C}=\mathrm{O})$ $\mathrm{COOH}), 1603$ («Amide-I», «Chlorin band»). UV-Vis $\left(\mathrm{CHCl}_{3}\right) \lambda_{\text {max }}$ $\mathrm{nm}$ : 664.0, 608.5, 557.0, 531.5, 502.0, 404.0. ${ }^{1} \mathrm{H}$ NMR (DMSO- $d_{6}$, $300 \mathrm{MHz}) \delta_{\mathrm{H}}$ ppm: 13.89 (1H, br.s, 13(1)-COOH), 12.13 (1H, br.s, 17(3)-COOH), $9.78\left(1 \mathrm{H}, \mathrm{s}, \mathrm{H}^{10}\right), 9.74\left(1 \mathrm{H}, \mathrm{s}, \mathrm{H}^{5}\right), 9.10\left(1 \mathrm{H}, \mathrm{s}, \mathrm{H}^{20}\right), 8.34$ $\left(1 \mathrm{H}, \mathrm{dd} J=18.0\right.$ and $\left.12.0 \mathrm{~Hz}, 3-\mathrm{CH}=\mathrm{CH}_{2}\right), 6.50(1 \mathrm{H}, \mathrm{d} J=18.0 \mathrm{~Hz}$, $\left.3-\mathrm{CH}=\mathrm{CH} H_{\text {nrans }}\right), 6.21\left(1 \mathrm{H}, \mathrm{d} J=12.0 \mathrm{~Hz}, 3-\mathrm{CH}=\mathrm{CH} H_{\mathrm{cis}}\right), 5.51(1 \mathrm{H}$, $\left.\mathrm{d} J=21.0 \mathrm{~Hz}, \mathrm{H}_{\mathrm{A}}^{15(1)}\right), 5.40\left(1 \mathrm{H}, \mathrm{d} J=21.0 \mathrm{~Hz}, \mathrm{H}^{15(1)}{ }_{\mathrm{B}}\right), 4.60(1 \mathrm{H}, \mathrm{q}$ $\left.J=9.0 \mathrm{~Hz}, \mathrm{H}^{18}\right), 4.36\left(1 \mathrm{H}\right.$, br.d $\left.J=9.0 \mathrm{~Hz} \mathrm{H}^{17}\right), 3.84(2 \mathrm{H}, \mathrm{q} J=9.0 \mathrm{~Hz}$, $\left.8-\mathrm{CH}_{2} \mathrm{CH}_{3}\right), 3.58\left(3 \mathrm{H}, \mathrm{s}, 12-\mathrm{CH}_{3}\right), 3.54\left(3 \mathrm{H}, \mathrm{s}, 2-\mathrm{CH}_{3}\right), 3.38(3 \mathrm{H}, \mathrm{s}$, 7- $\left.\mathrm{CH}_{3}\right), 2.36-2.21\left(2 \mathrm{H}, \mathrm{m}, 17-\mathrm{CH}_{2} \mathrm{CH}_{2} \mathrm{COOCH}_{3}\right), 2.21-2.06(2 \mathrm{H}$, m, $\left.17-\mathrm{CH}_{2} \mathrm{CH}_{2} \mathrm{COOCH}_{3}\right), 1.79-1.60\left(6 \mathrm{H}, \mathrm{m}, 18-\mathrm{CH}_{3}, 8-\mathrm{CH}_{2} \mathrm{CH}_{3}\right)$, $1.57-0.79\left(34 \mathrm{H}, \mathrm{m}, 15(2)-\mathrm{CON}\left(\mathrm{C}_{8} \mathrm{H}_{17}\right)_{2}\right),-1.64(1 \mathrm{H}$, br.s, I-NH), -1.94 (1H, br.s, III-NH).

Chlorin $e_{6}$ 15(2)-N-butylamide (13). $11.3 \mathrm{mg}$ (15 \%) of compound $\mathbf{1 3}$ as a dark green crystalline powder were obtained from $73.4 \mathrm{mg}$ of compound $\mathbf{8 . ~} \mathrm{m} / \mathrm{z}$ (ESI) calculated for $[\mathrm{MH}]^{+}$ $\left(\mathrm{C}_{38} \mathrm{H}_{45} \mathrm{~N}_{5} \mathrm{O}_{5}\right): 652.3$, found: 652.4. IR (KBr) $v_{\max } \mathrm{cm}^{-1}: 3410$ $(v(\mathrm{NHBu})), 3312(v(\mathrm{NH})), 2960,2928,2868,2735,2577,2561$ $\left(v(\mathrm{C}-\mathrm{H}) \mathrm{CH}_{3}, \mathrm{CH}_{2}\right), 1711(v(\mathrm{C}=\mathrm{O}) \mathrm{COOH}), 1632(«$ Amide-I»), 1599 («Chlorin band»). UV-Vis $\left(\mathrm{CHCl}_{3}\right) \lambda_{\max } \mathrm{nm}$ : 663.5, 608.0, 557.0, 531.5, 502.0, 403.5. ${ }^{1} \mathrm{H}$ NMR (DMSO- $\left.d_{6}, 300 \mathrm{MHz}\right) \delta_{\mathrm{H}} \mathrm{ppm}: 12.28$ $\left(2 \mathrm{H}\right.$, br.s, 13(1)-COOH and 17(3)-COOH), $9.81\left(1 \mathrm{H}, \mathrm{s}, \mathrm{H}^{10}\right), 9.73(1 \mathrm{H}$, s, $\left.\mathrm{H}^{5}\right), 9.16\left(1 \mathrm{H}, \mathrm{s}, \mathrm{H}^{20}\right), 9.24-9.08\left(1 \mathrm{H}, \mathrm{m}, 15^{2}-\left(\mathrm{CON} \mathrm{CC}_{4} \mathrm{H}_{9}\right)\right), 8.37$ $\left(1 \mathrm{H}\right.$, dd $J=18.0$ and $\left.12.0 \mathrm{~Hz}, 3-\mathrm{CH}=\mathrm{CH}_{2}\right), 6.48(1 \mathrm{H}, \mathrm{d} J=18.0 \mathrm{~Hz}$, $\left.3-\mathrm{CH}=\mathrm{CH}_{\mathrm{tran}}\right), 6.18\left(1 \mathrm{H}, \mathrm{d} J=12.0 \mathrm{~Hz}, 3-\mathrm{CH}=\mathrm{CH} H_{\text {cis }}\right), 5.69(1 \mathrm{H}$, $\left.\mathrm{d} J=21.0 \mathrm{~Hz}, \mathrm{H}^{15(1)}{ }_{\mathrm{A}}\right), 5.18-4.88\left(2 \mathrm{H}, \mathrm{m}, \mathrm{H}^{15(1)}{ }_{\mathrm{B}}, \mathrm{H}^{18}\right), 4.61(1 \mathrm{H}$, br.d $\left.J=9.0 \mathrm{~Hz}, \mathrm{H}^{17}\right), 3.85\left(2 \mathrm{H}, \mathrm{q} J=9.0 \mathrm{~Hz}, 8-\mathrm{CH}_{2} \mathrm{CH}_{3}\right), 3.58(3 \mathrm{H}, \mathrm{s}$, $\left.12-\mathrm{CH}_{3}\right), 3.54\left(3 \mathrm{H}, \mathrm{s}, 2-\mathrm{CH}_{3}\right), 3.38\left(3 \mathrm{H}, \mathrm{s}, 7-\mathrm{CH}_{3}\right), 2.34-2.09(4 \mathrm{H}$, $\left.\mathrm{m}, 17-\mathrm{CH}_{2} \mathrm{CH}_{2} \mathrm{COOCH}_{3}\right), 1.78-1.62\left(6 \mathrm{H}, \mathrm{m}, 18-\mathrm{CH}_{3}, 8-\mathrm{CH}_{2} \mathrm{CH}_{3}\right)$, $1.40-0.64\left(9 \mathrm{H}, \mathrm{m}, 15(2)-\mathrm{CONHC}_{4} \mathrm{H}_{9}\right),-1.94(1 \mathrm{H}$, br.s, I-NH), -2.50 (1H, br.s, III-NH).

Acknowledgments. This work was financially supported by the Russian Science Foundation (Project No. 16-1310367). The compounds synthesized were analyzed using the equipment of the "Chemistry" Center for Collective Use at the Institute of Chemistry of Komi Scientific Centre, Ural Branch of the Russian Academy of Sciences.

\section{References}

1. Mironov A.F. Ross. Khim. Zh. 1998, 42, 23-26 (in Russ.).

2. Zamilatskov I.A., Savinkina E.V., Volov A.N., Grigoriev M.S., Lonin I.S., Obolenskaya L.N., Ponomarev G.V., Koifman O.I., Kuzovlev A.S., Kuzmicheva G.M., Tsivadze A.Yu. Macroheterocycles 2012, 5, 308-314.

3. Nyman E.S., Hynninen P.H. J. Photochem. Photobiol., B 2004, 73(1-2), 1-28.

4. Ormond A.B., Freeman H.S. Materials 2013, 6, 817-840.

5. Nanobiotechnology: a Workshop (Rubin A.B., Ed.), Moscow: BINOM. Laboratory of Knowledge, 2011. 384 p.

6. Mazaletskaya L.I., Sheludchenko N.I., Khudyaeva I.S., Buravlev E.V., Belykh D.V., Chukicheva I.Yu. Macroheterocycles 2015, 4, 371-375.

7. Belykh D.V., Khudyaeva I.S., Buravlev E.V., Chukicheva I.Yu., Shevchenko O.G., Kutchin A.V. Russ. J. Org. Chem. 2017, 53, 610-614.

8. Belykh D.V., Buravlev E.V., Chukicheva I.Yu., Tarabukina I.S., Shevchenko O.G., Plyusnina S.N., Kutchin A.V. Russ. J. Bioorg. Chem. 2012, 38, 558-564.

9. Buravlev E.V., Belykh D.V., Chukicheva I.Yu., Tarabukina I.S., Shevchenko O.G., Kutchin A.V. Russ. J. Bioorg. Chem. 2013, 4, 434-437.

10. Belykh D.V., Kopylov E.A., Gruzdev I.V., Kuchin A.V. Russ. J. Org. Chem. 2010, 46, 577-585.

11. Dugin N.O., Zavialova M.G., Novikov R.A., Timofeev V.P., Misharin A.Yu., Ponomarev G.V. Macroheterocycles 2012, 2, 146-148.

12. Mal'shakova M.V., Belykh D.V., Hudyaev V.M., Kuchin A.V. Doklady Chemistry 2011, 436(2), 19-21.

13. Belykh D.V., Pushkareva E.I. Russ. J. Gen. Chem. 2011, 81, 1216-1221.

14. Belykh D.V., Mal'shakova M.V., Yudina Yu.A., Zavadskaya K.A., Khudyaev V.M., Kuchin A.V. Russ. Chem. Bull., Int. Ed. 2011, 60, 719-728.

15. Tulaeva L.A., Belykh D.V., Yakovleva N.M., Selkova I.A., Rocheva A.V., Kutchin A.V. Izv. Vyssh. Uchebn. Zaved., Khim. Khim. Tekhnol. 2006, 49(4), 82-87 (in Russ.). 